\title{
探讨维生素 $\mathbf{B}_{12}$ 研究史中的思政元素
}

张波，盛骞莹，张文清，赵怡，张凌怡，刘海燕，田佳，夏玮，钱俊红，王氢， 章弘扬, 胡坪 ${ }^{*}$

华东理工大学化学与分子工程学院, 上海 200237

摘要: 维生素 $B_{12}$ 的研究史蕴含了丰富的思政元素。本文通过探究维生素 $B_{12}$ 的发现、治疗恶性贫血机理、结构测定、 人工合成、生物合成中的关键人物、事件, 凝练出弘扬中医药学、用科学造福人类、团队合作、持之以恒、创新精 神等五大思政元素, 以期为相关专业课程中课程思政的开展带来有益的启迪。

关键词: 维生素B 12 ; 课程思政; 研究史

中图分类号: G64; O6

\section{Exploring the Ideological and Political Elements in the Research Process of Vitamin $B_{12}$}

Bo Zhang, Qianying Sheng, Wenqing Zhang, Yi Zhao, Lingyi Zhang, Haiyan Liu, Jia Tian, Wei Xia, Junhong Qian, Qing Wang, Hongyang Zhang, Ping Hu *

School of Chemistry and Molecular Engineering, East China University of Science and Technology, Shanghai 200237, China.

\begin{abstract}
Vitamin $B_{12}$ research process contains rich ideological and political elements. Based on exploring the discovery of vitamin $B_{12}$, the mechanism of treatment of pernicious anemia, $V_{12}$ structure determination, artificial synthesis and biosynthesis, five ideological and political elements were condensed in this article, such as promoting traditional Chinese medicine, benefiting mankind with science, teamwork, perseverance and innovative spirit. It is expected to bring beneficial enlightenment for the development of course ideological and political education in related professional courses.
\end{abstract}

Key Words: Vitamin $\mathrm{B}_{12} ; \quad$ Course ideology and politics; Research process

当今时代是信息化时代, 也是多元文化并存的时代, 如何帮助青年学生树立正确的价值观、道 德观, 是我们广大教师的责任。习近平总书记指出, “要用好课堂教学这个主渠道, 思想政治理论课 要坚持在改进中加强, 提升思想政治教育亲和力和针对性, 满足学生成长发展需求和期待, 其他各 门课程都要守好一段渠，种好责任田，使各类课程和思想政治理论课同向同行，形成协同效应 ${ }^{[1] 。 ” ~}$ 维生素 $\mathrm{B}_{12}$ (Vitamin $\mathrm{B}_{12}$, 以下简写为 $\mathrm{VB}_{12}$ ) 的发现、结构确立以及全合成的研究历史跨越生理学、微 生物学、分析化学、有机合成化学等多个领域, 包含丰富的科学精神, 其蕴含的思政元素能够给学 生带来有益和深刻的启迪。

收稿: 2021-07-07; 录用: 2021-08-11; 网络发表: 2021-09-10

“通讯作者, Email: huping@ecust.edu.cn

基金资助: 上海高校课程思政领航计划(重点改革领航学院); 化学类专业基础课程思政建设与实践(H20200507); 华东理工大学教师思政和师德 建设研究课题(YJ0109004) 


\section{$1 V B_{12}$ 的研究历史}

\section{$1.1 V B_{12}$ 的发现}

早在东汉末年, 我国名医华佗就曾用动物肝脏治疗贫血, 比西方早 1700 多年。元代《日用本草》 也有记载 “牛肝主治血虚萎黄, 虚劳贏瘦”。

19世纪 40 年代末, 英国伦敦的内科医生阿迪生报道了一种恶性贫血病, 患者表现出乏力、头晕、 腹胀、腹泻及舌炎、手足麻木、行走困难等, 备受痛苦折磨, 往往在3-5年内死亡。据统计, 当时每 年大约有 5 万名患者因恶性贫血去世。此后 70 多年间, 恶性贫血的病因始终无法找到, 病人处于绝望 的境地。1926年, 美国生理学家惠普尔报道, 通过大量实验发现吃生牛肝的狗贫血恢复最快, 这篇 报道对治疗恶性贫血的研究者来说犹如一道黑夜里的闪电, 哈佛医学院的乔治. 米诺特和威廉. 莫菲 受此启发, 让病人食用新鲜牛肝, 取得显著疗效 ${ }^{[2]}$, 但每天食用 1 斤左右的生牛肝实在难以下咽, 于 是他们联手哈佛大学的化学家艾德文. 科恩, 从牛肝中提取出浸膏, 疗效提高 50 倍, 病人不必每天 吃大量生肝。由于对治疗恶性贫血的突出贡献, 1934年惠普尔、米诺特、莫菲共同获得诺贝尔生理 或医学奖。1948年, 英美两国的研究人员几乎同时从牛肝浓缩物中分离出了一种红色晶体, 命名为 $\mathrm{VB}_{12}{ }^{[3]}$, 用 $\mathrm{VB}_{12}$ 治疗恶性贫血, 疗效比肝浓缩物提高一万倍。

后来的研究表明, $\mathrm{VB}_{12}$ 能够促进红细胞的发育和成熟、维持神经髓鞘的代谢与功能、提高叶酸 利用率, 另外, $\mathrm{VB}_{12}$ 还参与脱氧核糖核酸(DNA)的合成, 脂肪、碳水化合物及蛋白质的代谢, 促进 核酸与蛋白质的合成等, 对维护人体健康有重要作用。 $\mathrm{VB}_{12}$ 主要存在于肝脏、肉、蛋、奶等动物性 食品中, 人体自身无法合成, 只能通过食物摄取。人体吸收 $\mathrm{VB}_{12}$ 还需要通过胃壁细胞分泌的一种糖 蛋白, 胃黏膜受损、无胃酸或胃酸过少的人缺乏这种糖蛋白, 易患恶性贫血。

\section{$1.2 V_{12}$ 的结构测定}

$\mathrm{VB}_{12}$ 被发现之后的数十年间, 其化学结构一直未能被确定, 极大限制了对它的研究。1956年, 英国牛津大学的霍奇金利用X射线衍射法确定了 $\mathrm{VB}_{12}$ 的晶体结构, 1964年霍奇金及其同事因 “在测 定抗恶性贫血生化化合物 $\mathrm{VB}_{12}$ 中的杰出贡献” 获得诺贝尔化学奖。

$\mathrm{VB}_{12}$ 的分子量为 1355.38 , 是维生素家庭中分子量最大、结构最复杂、唯一含金属元素的成员, 也是除聚合物外分子量最大的天然产物。 $\mathrm{VB}_{12}$ (图1)是一个含有金属钴离子的类八面体大分子化合 物, 中心是平面钴卟啉环, 5,6-二甲基苯并咪唑(DMBI) 以 N-7 原子与钴离子相连成为 $\mathrm{VB}_{12}$ 分子的低位 配基, DMBI通过磷酸基与氨丙醇相连, 氨丙醇则与吡咯环上的丙酸侧链相连; 此外, 腺苷或甲基与

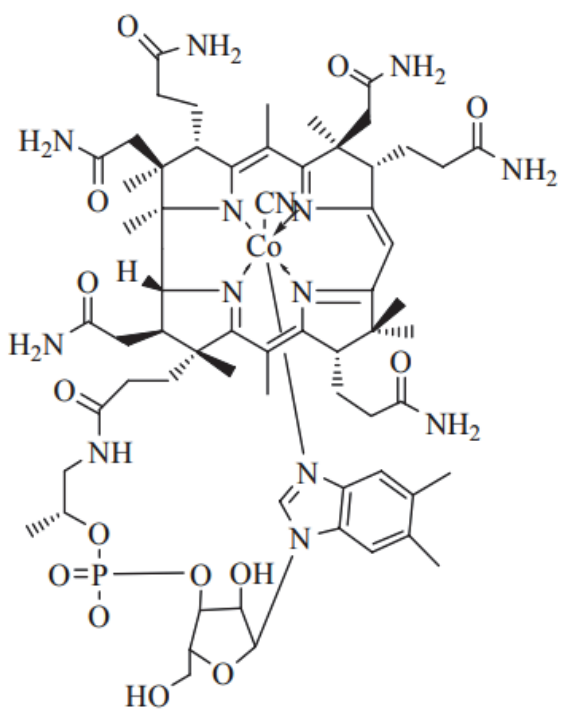

图1 VB12的化学结构 
钴离子相连组成 $\mathrm{VB}_{12}$ 分子的上位配基; 母核中还有 9 个不对称碳原子。咕啉环轴向上方的配基不同, 形成了不同类型的 $\mathrm{VB}_{12}$ 类物质。羟基与咕啉环中的钴离子相连形成羟基钴胺, 同样, 脱氧腺苷、甲 基、氯基与钴离子相连分别生成腺苷钴胺、甲基钴胺和氰钴胺等 ${ }^{[4]}$ 。 $\mathrm{VB}_{12}$ 的结构确定为其后的人工 合成和深入研究奠定了基础。

\section{$1.3 V_{12}$ 的合成}

起初, $\mathrm{VB}_{12}$ 只能从牛肝中提取, 所以价格昂贵, 而且供不应求, 这促使人们考虑人工合成 $\mathrm{VB}_{12}$ 。 $\mathrm{VB}_{12}$ 的合成堪称有机合成史上的杰作，代表了当时有机合成的最高水平。 $\mathrm{VB}_{12}$ 分子含有 181 个原子， 空间结构呈魔毡状, 结构非常复杂, 性质脆弱, 在强酸、强碱、高温作用下都会分解, 给合成带来 很大难度。 20 世纪 60 年代初, 哈佛大学的伍德沃德研究团队和瑞士联邦理工学院联合探索 $\mathrm{VB}_{12}$ 的全 合成研究。研究人员采取先合成 $\mathrm{VB}_{12}$ 的各个局部, 然后拼接起来的方法, 历经 11 年, 先后有来自 14 个国家的 110 多位研究人员参与了 $\mathrm{VB}_{12}$ 的合成研究工作, 在完成了 1100 多个独立的化学反应之后, 1972年, $\mathrm{VB}_{12}$ 的合成终于宣告完成。

在合成 $\mathrm{VB}_{12}$ 的过程中, 伍德沃德发现在加热和光照下进行环合反应能够得到不同的立体构型产 物。他和学生兼助手霍夫曼深入研究这一现象, 总结规律, 提出了分子轨道对称守恒理论, 即反应 物分子外层轨道对称一致时, 反应就易进行, 称为 “对称性允许”, 反应物分子外层轨道对称性不 一致时, 反应就不易进行, 称为 “对称性禁阻”。后来, 分子轨道对称守恒原理推广到无机、催化、 生化反应等许多重要领域, 成为微观化学反应动力学和量子化学应用的一个里程碑。由于分子轨道 对称守恒理论的提出, 1981年霍夫曼和另一位日本科学家共同获得诺贝尔化学奖。遗憾的是, 此时 伍德沃德已经去世两年了。

之后又经过多年的研究, 科学家们探索出 $\mathrm{VB}_{12}$ 的生物合成途径, 现在 $\mathrm{VB}_{12}$ 的工业生产主要通过 生物发酵实现, 我国生产 $\mathrm{VB}_{12}$ 的企业有宁夏多维、河北玉星、华北制药、河北华荣等, 产品销往世 界各地。

\section{$2 V_{12}$ 的研究历史带给我们的思政启示}

\section{1 中医药是我国古代科学和文化的瑰宝}

在人类与恶性贫血斗争的漫长道路上, 我国古代中医药典籍早已记载了治疗方法。中医药是我 国古代科学和文化的瑰宝, 在治疗疾病、保护健康方面起到重要积极的作用, 至今仍是不可替代的。 中医药典籍中的药方是历代名医治病救人实践智慧的结晶, 直到今天我们仍能从中获得宝贵的启示。 屠呦呦篮选抗疮药方时, 受东晋名医葛洪《肘后备急方》 “青蒿一握, 以水二升, 渍绞取汁, 尽服 之” 可治 “久㾏” 的启发, 提取青蒿素治疗症疾, 挽救了数百万人的生命。面对新冠疫情的肆虐, 我国医务人员将《伤寒杂病论》等古典医籍中的经典方剂融合, 推出 “三药三方”, 在疫病防治中 发挥了重要作用, 并被多个国家采用, 为世界抗疫贡献了中国力量。但是, 传统中药缺乏药理、有 效成分等方面的研究, 极大限制了中药的推广和应用。例如治疗小儿乳滞疳积、脾胃虚弱的名药 “王 氏保赤丸”，其说明书中 “不良反应” “禁忌” “注意事项” 均为 “尚不明确”, 这种情况在很多中 药制剂中都存在。如何将现代科技手段、分析方法与古老的中药制剂结合, 推动中药现代化, 让古 老的中药学焕发出新的生命力, 为人类健康做出更大贡献, 是青年一代的重任和使命。

\section{2 科技进步是提高人类健康水平的重要因素}

人类最终战胜恶性贫血的关键是通过科学研究找到病魔的克星- $\mathrm{VB}_{12}$, 证明了科学是挽救生 命、攻克病魔的良方。在人类发展历史中, 每当重大疾病肆虐之初, 人们往往束手无策, 无数生命 因疾病逝去, 但是, 最终人类利用科学得到战胜病魔的利器。众所周知, 二战后期青霉素的应用大 大减少了士兵的伤亡人数, 青霉素因其广谱的抗菌性、副作用小, 至今仍被广泛应用。2019年底至 今, 出现了全球性的新冠疫情大流行, 据世卫组织最新统计, 全球确认的新冠肺炎感染病例数超过 1 亿 6859 万, 死亡人数达 350 万, 由于许多国家缺乏可靠的死亡记录系统, 真实死亡人数可能要高出 
两到三倍。防控疫情的强力武器是疫苗, 面对肆虐的疫情, 各国科学家纷纷研制疫苗, 至今多国已 经研制出了疫苗并投入使用, 疫苗接种率高的国家如英国、以色列, 疫情得到明显控制, 再次证明 了科技进步对人类健康有多么重要! 通过以上生动的事例, 能够帮助学生树立起对科学的浓厚兴趣 以及用科学造福人类的远大理想。

\section{3 持之以恒是重要的科学精神}

人类研究 $\mathrm{VB}_{12}$ 的历史持续了一百多年, 经历了几代人的不解努力, 终于从 $\mathrm{VB}_{12}$ 的结构、功能、 合成等诸多方面逐渐揭开其面纱。从阿迪生报道恶性贫血之后的七十几年, 人们一直在探索治疗疾 病的方法, 但没有取得进展, 直到1926年, 美国科学家发现狗食用生肝贫血恢复最快, 同年该报道 被用于临床治疗恶性贫血患者, 疗效显著。但生肝为什么能够治疗恶性贫血, 原因尚不得知, 人们 推测牛肝中存在一种 “抗恶性贫血因子”。针对胃黏膜萎缩或胃切除的恶性贫血患者服用生肝无效 的现象, 1929年, 美国科学家卡斯特通过实验发现正常人的胃内物可以有效改善病人的贫血, 他由 此推断, 生肝中存在某种有效成分 “外因子”, 正常人的胃黏膜上存在用来吸收的 “内因子” (后来 被证实是胃壁细胞分泌的一种糖蛋白), 内外因子相结合, 才能治愈恶性贫血 ${ }^{[5]}$ 。内因子的发现解释 了 $\mathrm{VB}_{12}$ 的吸收模式, 而且也为恶性贫血的发病机理提供了关键线索。后来, 美国生物学家玛丽创建 了利用乳酸菌测定肝脏提取物中有效因子含量的方法, 基于此研究, 美国默克公司的研究团队在短 短 3 个月内, 分离出 $\mathrm{VB}_{12}$ 。7 年之后, $\mathrm{VB}_{12}$ 的结构被确定, 人们开始着手合成 $\mathrm{VB}_{12}, 1972$ 年, $\mathrm{VB}_{12}$ 实 验室合成成功。由于人工合成 $\mathrm{VB}_{12}$ 成本昂贵, 人们转向微生物发酵的方法制备。1993年, $\mathrm{VB}_{12}$ 的生 物合成途径被完整阐明, $\mathrm{VB}_{12}$ 已实现工业化生产。

$\mathrm{VB}_{12}$ 的研究历程(图2)清楚地向我们展示了人类是如何坚持不解、一步步抽丝剥茧般地探索大自 然的奥秘。正是几代科学家持之以恒的努力, 才使恶性贫血的发病原理和治疗方法浮出水面, 解除 了无数病人的痛苦。尤其在合成环节, 由于 $\mathrm{VB}_{12}$ 结构极其复杂, 且化学性质不稳定, 研究团队历时 11 年, 完成一千多个独立反应才获得成功。科学的道路从来都不是平坦的, 需要我们艰苦的付出, 持之以恒的努力, 才可能到达成功的彼岸。

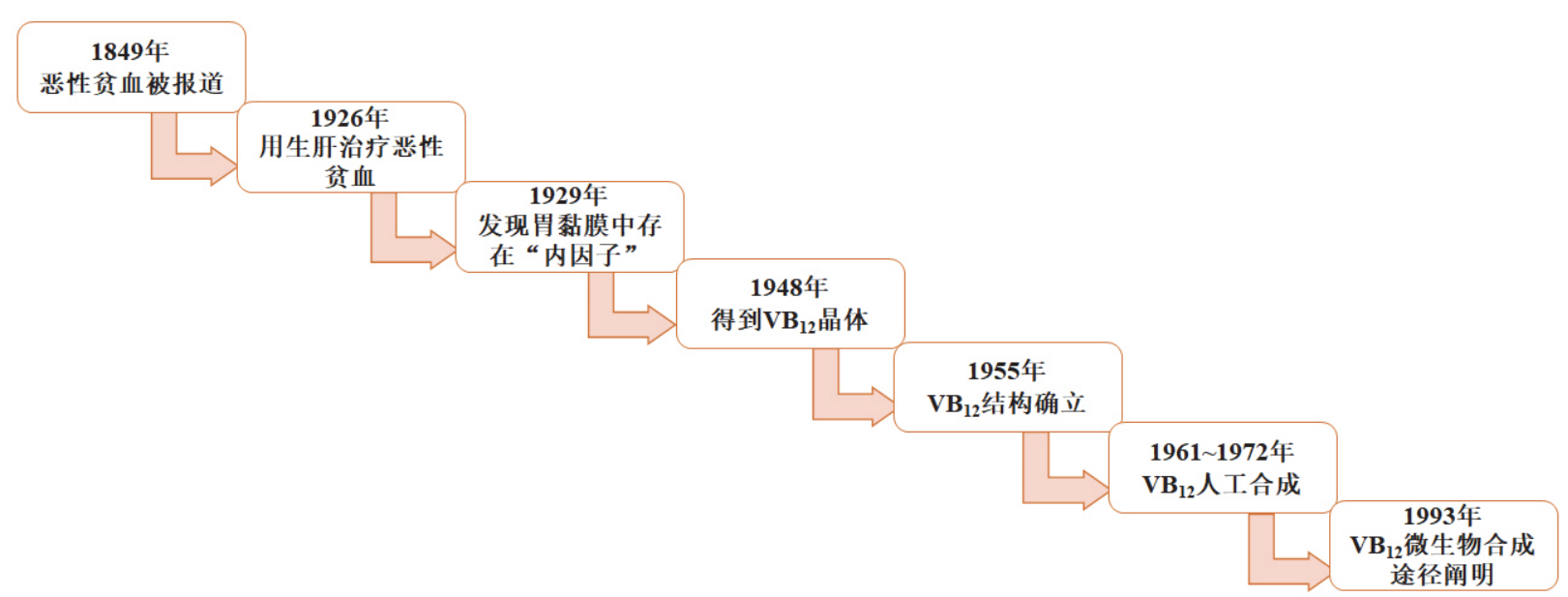

图2 VB 12 研究历程

\section{4 团队合作、协同攻关是成功的重要保证}

$\mathrm{VB}_{12}$ 的人工合成堪称有机合成史上的一座辉煌的里程碑。20世纪 60 年代初, 哈佛大学的沃德伍 德团队和瑞士联邦理工学院的艾申莫瑟团队合作研究 $\mathrm{VB}_{12}$ 的人工合成, 其关键是核心结构钴啉核的 合成, 如图3所示, 钴啉核可以通过片段 1 、片段 2 拼接而成。沃德伍德团队负责片段 1 的合成, 艾申 莫瑟团队负责片段 2 的合成, 之后再将两个片段汇聚合成以及完成尾链的合成 ${ }^{[6]}$ 。 


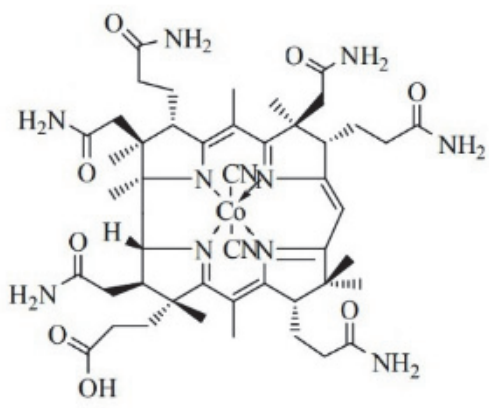

吓啉核

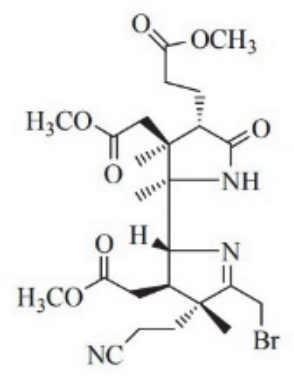

片段 1

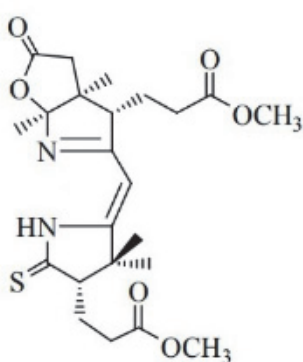

片段 2

图3 卟啉核的结构及合成

$\mathrm{VB}_{12}$ 的人工合成是一项极其艰苦的工作，先后有 110 多位科研人员参与其中，历时 11 年，大 家分工合作，协同攻关，克服很多困难，才得以完成有机合成史上这一经典之作。

当今社会分工越来越细, 很多工作需要人们合作努力才能完成。无论国与国之间, 还是人与人 之间, 合作是解决问题的良方。我们国家之所以能够迅速控制住来势汹汹的新冠疫情, 重要的原因 是政府及时下达了各项政策, 阻断疫情扩散渠道, 各省市医疗队赶赴支援疫区; 人们积极响应政府 号召, 全国人民上下同心, 社会各界全力以赴, 团结合作, 形成强大的力量, 这才使疫情局面及时 被扭转。反观很多国家, 民心涣散, 有令不行, 甚至各自为政, 导致疫情不断蔓延扩大。自然界中 雁群在天空中飞行呈 “V” 字型排列, 这种飞行方式比单独飞行能节省体能 $12 \%$, 可见良好的合作能 够产生 $1+1>2$ 的效果。青年学生要学会与人合作, 在工作和生活中构建良好和睦的合作关系, 这对 其一生是大有禆益的。

\section{5 创新是科学发展的动力}

在 $\mathrm{VB}_{12}$ 的合成过程中, 沃德伍德发现在己三烯的关环反应中, 光或热条件能够引发不同的立体 结构产物。他抓住这一现象深入研究, 在大量实验现象的基础上, 总结出多类周环反应的规律, 并 且和其学生霍夫曼从理论角度对现象进行分析, 1965年两人共同提出了分子轨道对称守恒原理, 又 称Woodward-Hoffman规则。分子轨道对称守恒理论把量子化学的分子轨道理论引入到化学反应体 系, 不但解释了已知的周环反应现象, 而且预言其后的许多反应产物, 它不仅能指导有机反应的研 究, 也能够应用于无机反应和催化反应等其他领域。分子轨道对称守恒理论使人们对反应动力学和 反应机理的认识深入到了物质微观结构的层次, 现已成为考查化学反应机理的主要理论方法, 被誉 为 “人类认识化学反应过程的发展道路上的里程碑” [7]。

当传统理论和方法无法应对新情况时, 必须考虑用新的理论和手段去解决问题。创新是科学不 断向前发展的动力, 也是人类文明前进的动力, 一个国家长久兴盛需要创新, 期待青年学子用勤劳 和智慧在创新的道路上留下扎实的足迹。

\section{3 结语}

笔者曾经在 “绪论” 一章的教学中讲述了 $\mathrm{VB}_{12}$ 的研究史, 其精彩的内容深深吸引了学生。 $\mathrm{VB}_{12}$ 的研究史是人类战胜恶性贫血的漫长历史, 也是人类探索大自然奥秘的历史。通过讲授这段历史, 我们专业课程教师可以把其中丰富的思政元素以 “春雨润物细无声” 的方式融入学生们的心中。青 年学生精力充沛、思想活跃, 他们肩负国家的希望, 是未来社会的建设者和主力军。通过探讨 $\mathrm{VB}_{12}$ 研究史中的思政内容, 可以帮助学生建立起把中医药发扬光大的信心, 引导学生树立合作、创新的 意识, 树立用科学造福人类的远大信念, 培养脚踏实地、持之以恒的工作作风, 尽师之责, 努力把 青年学生培养成为祖国的栋梁之才! 
[1] 习近平. 全国高校思想政治工作会议上的讲话. [2016-12-08]. http://www.xinhuanet.com//politics/2016-12/08/c_1120083340.htm

[2] Minot, G. R.; Murphy, W. P. J. Am. Med. Assoc. 1926, 87 (7), 470.

[3] Rickers, E. L.; Brink, N. G.; Koniuszy, F. R. Science 1948, 107 (2781), 396.

[4] 付炎, 李力更, 王于方, 王否, 史清文. 中草药, 2015, 46 (9), 1259.

[5] Elrod, J. M.; Karnad, A. B. Br. J. Haematol. 2003, 121 (3), 390.

[6] Khan, A. G.; Eswaran, S. V. Resonance 2003, 8 (6), 8.

[7] 王安琳. 科学技术与辩证法. 1994, 11 (4), 24. 